\title{
Supplementary Table S1.
}

Protein identification of 2D gel spots.

All spots were excised from the 2D gel of one wildtype contralateral hippocampus.

\begin{tabular}{|c|c|c|c|c|c|c|c|}
\hline \multicolumn{8}{|c|}{ SPOT 1 FIGURE 1} \\
\hline Name & $\begin{array}{c}\text { Swiss- } \\
\text { Prot } \\
\text { number }\end{array}$ & $\begin{array}{c}\text { Observed } \\
\mathbf{m} / \mathbf{z}\end{array}$ & $\begin{array}{c}\text { Peptide } \\
\text { Mass } \\
\text { calc. (Da) }\end{array}$ & $\begin{array}{c}\text { Peptide } \\
\text { Mass } \\
\text { theor. (Da) }\end{array}$ & $\begin{array}{l}\text { Delta } \\
\text { Mass }\end{array}$ & $\begin{array}{l}\text { Peptide } \\
\text { Score }^{1}\end{array}$ & Peptide sequence \\
\hline $\begin{array}{l}\text { tropomyosin alpha } \\
3 \text { chain }\end{array}$ & P21107 & $\begin{array}{l}401.72 \\
438.23 \\
447.74 \\
470.73 \\
574.31 \\
578.83 \\
591.78 \\
622.33 \\
642.88 \\
658.83 \\
467.26 \\
576.64 \\
\end{array}$ & $\begin{array}{c}801.44 \\
874.44 \\
893.46 \\
939.44 \\
1146.60 \\
1155.65 \\
1181.55 \\
1242.65 \\
1283.75 \\
1315.64 \\
1398.75 \\
1726.89 \\
\end{array}$ & $\begin{array}{c}801.43 \\
874.44 \\
893.46 \\
939.44 \\
1146.60 \\
1155.65 \\
1181.55 \\
1242.65 \\
1283.74 \\
1315.64 \\
1398.75 \\
1726.88 \\
\end{array}$ & $\begin{array}{l}0.00 \\
0.00 \\
0.00 \\
0.00 \\
0.00 \\
0.00 \\
0.00 \\
0.00 \\
0.00 \\
0.00 \\
0.00 \\
0.01\end{array}$ & $\begin{array}{l}16 \\
55 \\
44 \\
38 \\
47 \\
49 \\
22 \\
58 \\
57 \\
55 \\
41 \\
29\end{array}$ & $\begin{array}{l}\text { K.NVTNNLK.S } \\
\text { K.SLEAQAEK.Y } \\
\text { R.KYEEVAR.K } \\
\text { K.HIAEEADR.K } \\
\text { K.MOXELQEIQLK.E } \\
\text { K.LVIIEGDLER.T } \\
\text { K.EDKYEEEIK.I } \\
\text { R.IQLVEEELDR.A } \\
\text { R.KLVIIEGDLER.T } \\
\text { K.AADAEAEVASLNR.R } \\
\text { R.RIQLVEEELDR.A } \\
\text { R.IQLVEEELDRAQER.L }\end{array}$ \\
\hline 14-3-3 epsilon & P62259 & $\begin{array}{l}408.71 \\
454.27 \\
459.27 \\
497.25 \\
597.80 \\
603.33 \\
619.33 \\
628.80 \\
732.36 \\
\end{array}$ & $\begin{array}{c}815.41 \\
906.52 \\
916.52 \\
992.49 \\
1193.59 \\
1204.65 \\
1236.65 \\
1255.58 \\
1462.70 \\
\end{array}$ & $\begin{array}{c}815.41 \\
906.52 \\
916.52 \\
992.48 \\
1193.59 \\
1204.65 \\
1236.65 \\
1255.58 \\
1462.70 \\
\end{array}$ & $\begin{array}{l}0.00 \\
0.00 \\
0.00 \\
0.00 \\
0.00 \\
0.00 \\
0.00 \\
0.00 \\
0.00 \\
\end{array}$ & $\begin{array}{l}39 \\
38 \\
55 \\
24 \\
46 \\
46 \\
38 \\
59 \\
81\end{array}$ & $\begin{array}{l}\text { K.LAEQAER.Y } \\
\text { R.NLLSVAYK.N } \\
\text { R.IISSIEQK.E } \\
\text { R.QMoxVETELK.L } \\
\text { K.EAAENSLVAYK.A } \\
\text { K.DSTLIMoxQLLR.D } \\
\text { K.HLIPAANTGESK.V } \\
\text { R.YLAEFATGNDR.K } \\
\text { K.VAGMOxDVELTVEER.N }\end{array}$ \\
\hline $\begin{array}{l}\text { tropomyosin } 1 \\
\text { alpha chain }\end{array}$ & P58771 & $\begin{array}{l}438.23 \\
447.74 \\
574.31 \\
591.78 \\
593.84 \\
622.33 \\
438.93 \\
467.26 \\
576.64 \\
\end{array}$ & $\begin{array}{c}874.44 \\
893.46 \\
1146.60 \\
1181.55 \\
1185.66 \\
1242.65 \\
1313.76 \\
1398.75 \\
1726.89\end{array}$ & $\begin{array}{c}874.44 \\
893.46 \\
1146.60 \\
1181.55 \\
1185.66 \\
1242.65 \\
1313.76 \\
1398.75 \\
1726.88 \\
\end{array}$ & $\begin{array}{l}0.00 \\
0.00 \\
0.00 \\
0.00 \\
0.00 \\
0.00 \\
0.00 \\
0.00 \\
0.01\end{array}$ & $\begin{array}{l}55 \\
44 \\
47 \\
22 \\
50 \\
58 \\
36 \\
41 \\
29\end{array}$ & $\begin{array}{l}\text { K.SLEAQAEK.Y } \\
\text { R.KYEEVAR.K } \\
\text { K.MoXEIQEIQLK.E } \\
\text { K.EDKYEEEIK.V } \\
\text { K.LVIIESDLER.A } \\
\text { R.IQLVEEELDR.A } \\
\text { R.KLVIIESDLER.A } \\
\text { R.RIQLVEEELDR.A } \\
\text { R.IQLVEEELDRAQER.L }\end{array}$ \\
\hline
\end{tabular}




\begin{tabular}{|c|c|c|c|c|c|c|c|}
\hline 14-3-3 gamma & P61982 & $\begin{array}{l}408.71 \\
452.26 \\
454.27 \\
540.78 \\
603.33\end{array}$ & $\begin{array}{c}815.41 \\
902.51 \\
906.52 \\
1079.55 \\
1204.65\end{array}$ & $\begin{array}{c}815.41 \\
902.51 \\
906.52 \\
1079.55 \\
1204.65\end{array}$ & $\begin{array}{l}0.00 \\
0.00 \\
0.00 \\
0.00 \\
0.00\end{array}$ & $\begin{array}{l}39 \\
37 \\
38 \\
28 \\
46\end{array}$ & $\begin{array}{l}\text { R.LAEQAER.Y } \\
\text { R.VISSIEQK.T } \\
\text { R.NLLSVAYK.N } \\
\text { R.YLAEVATGEK.R } \\
\text { K.DSTLIMoxQLLR.D }\end{array}$ \\
\hline 14-3-3 sigma & O70456 & $\begin{array}{l}408.71 \\
452.26 \\
454.27 \\
603.33\end{array}$ & $\begin{array}{c}815.41 \\
902.51 \\
906.52 \\
1204.65\end{array}$ & $\begin{array}{c}815.41 \\
902.51 \\
906.52 \\
1204.65\end{array}$ & $\begin{array}{l}0.00 \\
0.00 \\
0.00 \\
0.00\end{array}$ & $\begin{array}{l}39 \\
37 \\
38 \\
46\end{array}$ & $\begin{array}{l}\text { K.LAEQAER.Y } \\
\text { R.VLSSIEQK.S } \\
\text { R.NLLSVAYK.N } \\
\text { K.DSTLIMoxQLLR.D }\end{array}$ \\
\hline \multicolumn{8}{|c|}{ SPOT 2 FIGURE 1} \\
\hline Name & $\begin{array}{c}\text { Swiss- } \\
\text { Prot } \\
\text { number }\end{array}$ & $\begin{array}{c}\text { Observed } \\
\mathbf{m} / \mathbf{z}\end{array}$ & $\begin{array}{c}\text { Peptide } \\
\text { Mass } \\
\text { calc. (Da) }\end{array}$ & $\begin{array}{c}\text { Peptide } \\
\text { Mass } \\
\text { theor. (Da) } \\
\end{array}$ & $\begin{array}{l}\text { Delta } \\
\text { Mass }\end{array}$ & $\begin{array}{l}\text { Peptide } \\
\text { Score }{ }^{1}\end{array}$ & Peptide sequence \\
\hline $14-3-3$ eta & P68510 & $\begin{array}{l}412.21 \\
452.26 \\
454.27 \\
533.77 \\
603.33 \\
634.31 \\
793.89\end{array}$ & $\begin{array}{c}822.41 \\
902.51 \\
906.52 \\
1065.54 \\
1204.65 \\
1266.61 \\
1585.76 \\
\end{array}$ & $\begin{array}{c}822.41 \\
902.51 \\
906.52 \\
1065.54 \\
1204.65 \\
1266.61 \\
1585.76 \\
\end{array}$ & $\begin{array}{l}0.00 \\
0.00 \\
0.00 \\
0.00 \\
0.00 \\
0.00 \\
0.00\end{array}$ & $\begin{array}{l}33 \\
48 \\
31 \\
51 \\
42 \\
73 \\
73\end{array}$ & $\begin{array}{l}\text { K.EAFEISK.E } \\
\text { R.VISSIEQK.T } \\
\text { R.NLLSVAYK.N } \\
\text { R.YLAEVASGEK.K } \\
\text { K.DSTLIMoxQLLR.D } \\
\text { K.NSVVEASEAAYK.E } \\
\text { K.AVTELNEPLSNEDR.N }\end{array}$ \\
\hline 14-3-3 zeta/delta & P63101 & $\begin{array}{l}445.25 \\
454.27 \\
509.28 \\
603.33 \\
640.33 \\
665.85 \\
774.86\end{array}$ & $\begin{array}{c}888.49 \\
906.52 \\
1016.55 \\
1204.65 \\
1278.65 \\
1329.69 \\
1547.71 \\
\end{array}$ & $\begin{array}{c}888.49 \\
906.52 \\
1016.55 \\
1204.65 \\
1278.65 \\
1329.69 \\
1547.71 \\
\end{array}$ & $\begin{array}{l}0.00 \\
0.00 \\
0.00 \\
0.00 \\
0.00 \\
0.00 \\
0.00\end{array}$ & $\begin{array}{l}46 \\
31 \\
26 \\
42 \\
62 \\
50 \\
84\end{array}$ & $\begin{array}{l}\text { R.VVSSIEQK.T } \\
\text { R.NLLSVAYK.N } \\
\text { R.EKIETELR.D } \\
\text { K.DSTLIMoxQLLR.D } \\
\text { R.YLAEVAAGDDKK.G } \\
\text { K.FLIPNASQPESK.V } \\
\text { K.SVTEQGAELSNEER.N }\end{array}$ \\
\hline $\begin{array}{l}\text { proteasome } \\
\text { subunit alpha } \\
\text { type } 3\end{array}$ & O70435 & $\begin{array}{l}426.70 \\
475.25 \\
495.25 \\
565.78 \\
576.81 \\
609.33 \\
491.28\end{array}$ & $\begin{array}{c}851.38 \\
948.49 \\
988.48 \\
1129.55 \\
1151.60 \\
1216.64 \\
1470.81 \\
\end{array}$ & $\begin{array}{c}851.38 \\
948.49 \\
988.48 \\
1129.55 \\
1151.60 \\
1216.64 \\
1470.81 \\
\end{array}$ & $\begin{array}{l}0.00 \\
0.00 \\
0.00 \\
0.00 \\
0.00 \\
0.00 \\
0.00\end{array}$ & $\begin{array}{l}25 \\
42 \\
22 \\
48 \\
41 \\
69 \\
14\end{array}$ & $\begin{array}{l}\text { R.EEASNFR.S } \\
\text { K.DGVVFGVEK.L } \\
\text { K.DIREEAEK.Y } \\
\text { R.VFQVEYAMoxK.A } \\
\text { R.SNFGYNIPLK.H } \\
\text { K.AVENSSTAIGIR.C } \\
\text { K.IIYIVHDEVKDK.A }\end{array}$ \\
\hline 14-3-3 sigma & O70456 & $\begin{array}{l}452.26 \\
454.27 \\
603.33\end{array}$ & $\begin{array}{c}902.51 \\
906.52 \\
1204.65\end{array}$ & $\begin{array}{c}902.51 \\
906.52 \\
1204.65\end{array}$ & $\begin{array}{l}0.00 \\
0.00 \\
0.00\end{array}$ & $\begin{array}{l}48 \\
31 \\
42\end{array}$ & $\begin{array}{l}\text { R.VISSIEQK.T } \\
\text { R.NLLSVAYK.N } \\
\text { K.DSTLIMoxQLLR.D }\end{array}$ \\
\hline
\end{tabular}




\begin{tabular}{|c|c|c|c|c|c|c|c|}
\hline \multicolumn{8}{|c|}{ SPOT 3 FIGURE 1} \\
\hline Name & $\begin{array}{c}\text { Swiss- } \\
\text { Prot } \\
\text { number }\end{array}$ & $\begin{array}{c}\text { Observed } \\
\mathbf{m} / \mathbf{z}\end{array}$ & $\begin{array}{c}\text { Peptide } \\
\text { Mass } \\
\text { calc. (Da) }\end{array}$ & $\begin{array}{c}\text { Peptide } \\
\text { Mass } \\
\text { theor. (Da) }\end{array}$ & $\begin{array}{l}\text { Delta } \\
\text { Mass }\end{array}$ & $\begin{array}{l}\text { Peptide } \\
\text { Score }^{1}\end{array}$ & Peptide sequence \\
\hline $\begin{array}{l}\text { F-actin capping } \\
\text { protein beta } \\
\text { subunit }\end{array}$ & P47757 & $\begin{array}{l}476.78 \\
497.23 \\
508.30 \\
554.83 \\
586.30 \\
616.27 \\
677.33 \\
767.85 \\
784.87 \\
562.60 \\
848.92 \\
576.52 \\
\end{array}$ & $\begin{array}{c}951.54 \\
992.45 \\
1014.58 \\
1107.64 \\
1170.59 \\
1230.53 \\
1352.64 \\
1533.68 \\
1567.73 \\
1684.79 \\
1695.82 \\
2302.03 \\
\end{array}$ & $\begin{array}{c}951.54 \\
992.45 \\
1014.58 \\
1107.64 \\
1170.59 \\
1230.53 \\
1352.64 \\
1533.68 \\
1567.73 \\
1684.79 \\
1695.82 \\
2302.03 \\
\end{array}$ & $\begin{array}{l}0.00 \\
0.00 \\
0.00 \\
0.00 \\
0.00 \\
0.00 \\
0.00 \\
0.00 \\
0.00 \\
0.00 \\
0.00 \\
0.00\end{array}$ & $\begin{array}{c}36 \\
47 \\
42 \\
36 \\
59 \\
37 \\
95 \\
60 \\
79 \\
42 \\
128 \\
37 \\
\end{array}$ & $\begin{array}{l}\text { R.LPPQQIEK.N } \\
\text { R.LVEDMoxENK.I } \\
\text { K.TKDIVNGLR.S } \\
\text { R.RLPPQQIEK.N } \\
\text { R.STLNEIYFGK.T } \\
\text { K.DYLLCDYNR.D } \\
\text { K.SGSGTMOXNLGGSLTR.Q } \\
\text { K.YDPPLEDGAMoxPSAR.L } \\
\text { K.LEVEANNAFDQYR.D } \\
\text { K.GCWDSIHVVEVQEK.S } \\
\text { R.KLEVEANNAFDQYR.D } \\
\text { R.QMOXEKDETVSDCSPHIANIGR.L }\end{array}$ \\
\hline 14-3-3 epsilon & P62259 & $\begin{array}{l}454.27 \\
459.27 \\
497.25 \\
597.80 \\
603.33 \\
619.33 \\
628.80 \\
732.36\end{array}$ & $\begin{array}{c}906.52 \\
916.52 \\
992.49 \\
1193.59 \\
1204.65 \\
1236.65 \\
1255.59 \\
1462.70\end{array}$ & $\begin{array}{c}906.52 \\
916.52 \\
992.48 \\
1193.59 \\
1204.65 \\
1236.65 \\
1255.58 \\
1462.70\end{array}$ & $\begin{array}{l}0.00 \\
0.00 \\
0.00 \\
0.00 \\
0.00 \\
0.00 \\
0.00 \\
0.00\end{array}$ & $\begin{array}{l}30 \\
48 \\
23 \\
44 \\
42 \\
46 \\
56 \\
94\end{array}$ & $\begin{array}{l}\text { R.NLLSVAYK.N } \\
\text { R.IISSIEQK.E } \\
\text { R.QMoxVETELK.L } \\
\text { K.EAAENSLVAYK.A } \\
\text { K.DSTLIMoxQLLR.D } \\
\text { K.HLIPAANTGESK.V } \\
\text { R.YLAEFATGNDR.K } \\
\text { K.VAGMoxDVELTVEER.N }\end{array}$ \\
\hline $\begin{array}{l}\text { purine nucleoside } \\
\text { phosporylase }\end{array}$ & P23492 & $\begin{array}{l}430.24 \\
563.32 \\
628.30 \\
\end{array}$ & $\begin{array}{c}858.46 \\
1124.62 \\
1254.58 \\
\end{array}$ & $\begin{array}{c}858.46 \\
1124.62 \\
1254.58 \\
\end{array}$ & $\begin{array}{l}0.00 \\
0.00 \\
0.00\end{array}$ & $\begin{array}{l}53 \\
55 \\
36\end{array}$ & $\begin{array}{l}\text { K.AAAQTLER.F } \\
\text { R.VFGFSLITNK.V } \\
\text { K.VVMoxDYENLEK.A }\end{array}$ \\
\hline \multicolumn{8}{|c|}{ SPOT 1 FIGURE 3} \\
\hline Name & $\begin{array}{c}\text { Swiss- } \\
\text { Prot } \\
\text { number }\end{array}$ & $\begin{array}{c}\text { Observed } \\
\mathbf{m} / \mathbf{z}\end{array}$ & $\begin{array}{l}\text { Peptide } \\
\text { Mass } \\
\text { calc. (Da) }\end{array}$ & $\begin{array}{c}\text { Peptide } \\
\text { Mass } \\
\text { theor. (Da) }\end{array}$ & $\begin{array}{l}\text { Delta } \\
\text { Mass }\end{array}$ & $\begin{array}{l}\text { Peptide } \\
\text { Score }^{1}\end{array}$ & Peptide sequence \\
\hline $\begin{array}{l}\text { proteasome } \\
\text { subunit alpha type } \\
5\end{array}$ & Q9Z2U1 & $\begin{array}{l}532.26 \\
724.37 \\
654.66\end{array}$ & $\begin{array}{l}1062.51 \\
1446.73 \\
1960.95\end{array}$ & $\begin{array}{l}1062.51 \\
1446.73 \\
1960.95\end{array}$ & $\begin{array}{l}0.00 \\
0.00 \\
0.00\end{array}$ & $\begin{array}{l}45 \\
51 \\
41\end{array}$ & $\begin{array}{l}\text { R.GVNTFSPEGR.L } \\
\text { R.ITSPLMoxEPSSIEK.I } \\
\text { R.AIGSASEGAQSSLQEVYHK.S }\end{array}$ \\
\hline 14-3-3 zeta/delta & P63101 & $\begin{array}{l}445.25 \\
454.27 \\
\end{array}$ & $\begin{array}{l}888.49 \\
906.52 \\
\end{array}$ & $\begin{array}{l}888.49 \\
906.52 \\
\end{array}$ & $\begin{array}{l}0.00 \\
0.00\end{array}$ & $\begin{array}{l}44 \\
30\end{array}$ & $\begin{array}{l}\text { R.VVSSIEQK.T } \\
\text { R.NLLSVAYK.N }\end{array}$ \\
\hline $\begin{array}{l}14-3-3 \text { beta/alpha } \\
\text { 14-3-3 epsilon } \\
14-3-3 \text { eta } \\
14-3-3 \text { gamma }\end{array}$ & $\begin{array}{c}\text { Q9CQV8 } \\
\text { P62259 } \\
\text { P68510 } \\
\text { P61982 }\end{array}$ & $\begin{array}{l}452.26 \\
454.27\end{array}$ & $\begin{array}{l}902.51 \\
906.52\end{array}$ & $\begin{array}{l}902.51 \\
906.52\end{array}$ & $\begin{array}{l}0.00 \\
0.00\end{array}$ & $\begin{array}{l}36 \\
30\end{array}$ & $\begin{array}{l}\text { R.VISSIEQK.T } \\
\text { R.NLLSVAYK.N }\end{array}$ \\
\hline
\end{tabular}


${ }^{1}$ Score is $-10^{*} \log (\mathrm{P})$, where $\mathrm{P}$ is the probability that the observed match is a random event. Individual ions scores $>22$ indicate identity or extensive homology $(\mathrm{p}<0.01)$. 